\title{
Concise Commentary: Spaced Out-Reducing the Relapse Risk in IBD Patients by Lengthening Dosing Intervals of Anti-TNFs
}

\author{
Pauline Rivière $^{1} \cdot$ David Laharie ${ }^{1}$
}

Published online: 3 February 2020

(c) Springer Science+Business Media, LLC, part of Springer Nature 2020

Following the revolution that started twenty years ago with the introduction of tumor necrosis factor antibodies (TNFs) for treating inflammatory bowel disease (IBD), the current challenge for physicians is to determine how long patients should be treated regarding the risks of these agents [1]. In Crohn's disease (CD), stopping TNFs was associated with a $50 \%$ risk of relapse in the following year [2]. Thus, increasing the interval between anti-TNF infusions or injections to rather than stopping therapy completely was proposed as an intermediate solution bridging drug withdrawal and using accepted dosing intervals. To date, little data have been published to support this strategy; the last European Crohn's and Colitis Organization guidelines could not reach consensus regarding the continuation or withdrawal of anti-TNFs in CD patients after achieving long-term remission [3].

In this issue of Digestive Diseases and Sciences, Torres et al. [4] retrospectively investigated the clinical outcomes of 85 IBD patients in clinical remission receiving infliximab or adalimumab at longer intervals than as recommended by regulatory bodies, every 10-12 weeks for infliximab and 3 weeks for adalimumab. The success of this dosing interval lengthening (DIL) was defined as the absence of clinical relapse, return to the conventional dosing schedule, dose escalation, change in biological, introduction of conventional immunosuppressants, prescription of a course of systemic corticosteroids, or surgery at the end of follow-up. Overall, 59\% experienced success of DIL; the median time for treatment failure was 16 months.

This is the largest published cohort so far on anti-TNF therapy de-escalation DIL, providing noteworthy data on a matter of importance regarding patients' quality of life

David Laharie

david.laharie@chu-bordeaux.fr

1 CHU de Bordeaux, Hôpital Haut-Lévêque, Service d'Hépato-Gastroentérologie Et Oncologie Digestive, Université de Bordeaux, 33000 Bordeaux, France and healthcare costs [5]. Yet, it seems difficult to use these results to support DIL in clinical practice.

The dose interval of anti-TNFs was determined based on physician's decisions without a pre-established strategy, leading to a possible selection bias toward a population with less severe disease. Although remnant inflammatory activity is predictive of relapse after stopping therapy [2], no recent endoscopic evaluation was available for the majority of patients, with four patients undergoing DIL despite the presence of active endoscopic lesions. Moreover, Torres et al. show that the success of DIL is associated with prior lower C-reactive protein values. The severity of disease relapse should also be discussed with the patient before DIL; in this study, four patients needed rescue treatment after non-response to reducing the dose interval and one patient required surgery. Drug concentrations prior and after DIL were available only for a small subset of patients, hampering the possibilities to draw results from these values.

Regarding the high risk of relapse and its potential adverse consequences, it appears to be obligatory to assess the biological and endoscopic activities of the disease in order to prove deep remission before instituting DIL with TNFs. The impact of therapeutic drug monitoring for guiding the physician for this setting is being explored by an ongoing trial (clinicaltrials.gov NCT03841942), which is expected to yield valuable data regarding patient selection for DIL which in turn should improve outcomes.

\section{Compliance with Ethical Standards}

Conflict of interest PR declares consultancy fees from Abbvie and Janssen. DL declares counseling, boards, transports or fees from Abbvie, Biogaran, Biogen, Ferring, HAC-pharma, Janssen, MSD, Novartis, Pfizer, Prometheus, Roche, Takeda, Theradiag, Tillots. 


\section{References}

1. 1. Annese V, Beaugerie L, Egan L, Biancone L, Bolling C, Brandts $\mathrm{C}$, et al. European evidence-based consensus: Inflammatory bowel disease and malignancies. J Crohns Colitis. 2015;9:945-65.

2. Louis E, Mary J-Y, Vernier-Massouille G, Grimaud J-C, Bouhnik $\mathrm{Y}$, Laharie D, et al. Maintenance of remission among patients with Crohn's disease on antimetabolite therapy after infliximab therapy is stopped. Gastroenterology. 2012;142:63-70.

3. Torres J, Bonovas S, Doherty G, Kucharzik T, Gisbert JP, Raine $\mathrm{T}$, et al. ECCO guidelines on therapeutics in Crohn's disease: medical treatment. J Crohns Colitis. 2020;14:4-22.

4. Torres P, Cañete F, Nuñez L, Aguilar A, Mesonero F, Calafat $\mathrm{M}$, et al. Spacing the administration interval of anti-TNF agents: avalid strategy for patients with inflammatory bowel disease? Dig Dis Sci. (Epub ahead of print). https://doi.org/10.1007/s1062 0-019-06010-w

5. Valk ME van der, Mangen M-JJ, Leenders M, Dijkstra G, Bodegraven AA van, Fidder HH, et al. Healthcare costs of inflammatory bowel disease have shifted from hospitalisation and surgery towards anti-TNF $\alpha$ therapy: results from the COIN study. Gut. 2014;63:72-79.

Publisher's Note Springer Nature remains neutral with regard to jurisdictional claims in published maps and institutional affiliations. 